\title{
Volume Rather Than Flow Incentive Spirometry Is Effective in Improving Chest Wall Expansion and Abdominal Displacement Using Optoelectronic Plethysmography
}

\author{
Denise de Moraes Paisani PhD, Adriana Claudia Lunardi PhD, \\ Cibele Cristine Berto Marques da Silva MSc, Desiderio Cano Porras, \\ Clarice Tanaka PhD, and Celso Ricardo Fernandes Carvalho PhD
}

\begin{abstract}
BACKGROUND: Incentive spirometers are widely used in clinical practice and classified as floworiented (FIS) and volume-oriented (VIS). Until recently the respiratory inductive plethysmography used to evaluate the effects of incentive spirometry on chest wall mechanics presented limitations, which may explain why the impact of VIS and FIS remains poorly known. OBJECTIVE: To compare the effects of VIS and FIS on thoracoabdominal mechanics and respiratory muscle activity in healthy volunteers. METHODS: This cross-sectional trial assessed 20 subjects (12 female, ages 20-40 years, body mass index $20-30 \mathrm{~kg} / \mathrm{m}^{2}$ ). All subjects performed 8 quiet breaths and 8 deep breaths with FIS and VIS, in a randomized order. We measured thoracoabdominal chest wall, upper and lower rib-cage, and abdominal volumes with optoelectronic plethysmography, and the muscle activity of the sternocleidomastoid and superior and inferior intercostal muscles with electromyography. RESULTS: VIS increased chest wall volume more than did FIS $(P=.007)$ and induced a larger increase in the upper and lower rib-cages and abdomen $(156 \%, 91 \%$, and $151 \%$, respectively, $P<.001)$. By contrast, FIS induced more activity in the accessory muscles of respiration than did VIS $(P<.001)$. CONCLUSIONS: VIS promotes a greater increase in chest wall volume, with a larger abdominal contribution and lower respiratory muscle activity, than does FIS in healthy adults. Key words: breathing exercises; physical therapy modalities; electromyography; biomechanics; incentive spirometry; chest wall; respiratory mechanics; lung volume measurements. [Respir Care 2013;58(8):1360-1366. (C) 2013 Daedalus Enterprises]
\end{abstract}

\section{Introduction}

Several clinical situations are often followed by respiratory impairment, particularly in patients undergoing thoracic $^{1}$ and abdominal surgeries ${ }^{2}$ or during prolonged bed

The authors are affiliated with the Department of Physical Therapy, School of Medicine, University of São Paulo, São Paulo, Brazil.

This research was partly supported by Conselho Nacional de Desenvolvimento Científico e Tecnológico and Fundação de Amparo à Pesquisa do Estado de São Paulo, Brazil.

Correspondence: Celso Ricardo Fernandes Carvalho $\mathrm{PhD}$, Department Physical Therapy, University of São Paulo, Rua Cipotanea, 51 Cidade Universitária, São Paulo, Brazil 05360160. E-mail: cscarval@usp.br.

DOI: $10.4187 /$ respcare.02037 rest. ${ }^{3,4}$ In such cases, respiratory impairment includes a reduction in both chest wall volume and bronchial capacity, which may result in respiratory infection. Incentive spirometry can be used to encourage deeper breaths and provide increased respiratory capacity, thus reversing alveolar collapse and improving oxygenation. Incentive spirometer has been used for the prophylaxis and treatment of pulmonary complications during abdominal, ${ }^{5-7}$ cardiac, ${ }^{8}$ and thoracic surgeries. ${ }^{9}$

There are 2 types of incentive spirometry: flow-oriented (FIS) and volume-oriented (VIS). Both provide visual feedback aimed at improving pulmonary expansion, and it seems that FIS demands higher inspiratory flow than does VIS.7 Despite the widespread use of incentive spirometry, recent systematic reviews suggest that their benefits are controversial, based on the questionable methodological quality of previous trials. ${ }^{5}$ In addition, few studies have evaluated 
the effect of FIS and VIS on thoracoabdominal mechanics, which is necessary to determine the use of these devices based on the therapeutic goals.

Parreira et $\mathrm{al}^{10}$ and Tomich et al ${ }^{11}$ showed that VIS induced a higher pulmonary volume than FIS, although both devices induced similar displacement of the abdominal and thoracic compartments. In addition, they showed that FIS induced a higher breathing frequency and accessory respiratory muscle activity than did VIS. Nevertheless, subjects in both studies were assessed in a dorsal (supine) position, which restricts chest wall expansion and reduces the diaphragm's capacity to generate strength. ${ }^{12}$ In addition, thoracoabdominal mechanics were measured using respiratory inductive plethysmography, a technique that evaluates pulmonary volume using a 2-compartment analysis that does not have an accurate calibration method.

Recently, optoelectronic plethysmography was developed to analyze chest wall kinematics, using a 3-compartment analysis that measures pulmonary volume and thoracoabdominal synchrony, and makes it possible to evaluate the subject in any position with the simultaneous analysis of respiratory muscle activity by using surface electromyography (EMG). ${ }^{12-14}$ Our hypothesis is that the similar results between FIS and VIS observed in previous studies are the result of using an inaccurate instrument to evaluate thoracoabdominal mechanics and an inappropriate subject position. In the present study we compared the effect of VIS and FIS on thoracoabdominal mechanics and respiratory muscle activity evaluated by optoelectronic plethysmography in healthy volunteers in a seated position.

\section{Methods}

\section{Design}

This cross-sectional trial was performed in healthy volunteers. The study was approved by the hospital's ethics committee (protocol 150/11), and all subjects provided written informed consent.

\section{Subjects}

Adults age 20-40 years and with a body mass index between 20 and $30 \mathrm{~kg} / \mathrm{m}^{2}$ were evaluated. The exclusion criteria were deformity of the chest wall or spine, $\mathrm{FEV}_{1}$ or FVC $<80 \%$ predicted or $\mathrm{FEF}_{25-75 \%}<60 \%$ predicted, cardiac disease, and current smoking. All subjects were unfamiliar with the incentive spirometer and reported never before having used this equipment. Instructions about the use of the devices were given just before the measurements. Subjects were evaluated between April 2011 and January 2012. Two subjects were excluded before evaluations due to a diagnosis of asthma.

\section{QUICK LOOK}

\section{Current knowledge}

Both types of incentive spirometer (flow-oriented and volume-oriented) provide visual feedback to improve pulmonary expansion. There have been few comparisons of flow versus volume incentive spirometers.

\section{What this paper contributes to our knowledge}

In healthy adults, volume incentive spirometer promoted a greater increase in chest wall volume, with a larger abdominal contribution, and lower respiratory muscle activity, than did flow incentive spirometer.

\section{Study Protocol}

After signing the informed consent, subjects performed spirometry according to the European Respiratory Society/ American Thoracic Society protocol, ${ }^{15}$ followed by optoelectronic plethysmography of thoracoabdominal mechanics, at rest and during FIS and VIS.

The assessment of thoracoabdominal kinematics and inspiratory muscle activity was performed during quiet breathing, followed by either VIS or FIS, guided by a respiratory therapist. The order of use (VIS or FIS) was randomly determined and placed in sealed envelopes that were numbered sequentially by an independent researcher. All subjects performed 8 quiet breaths, followed by 8 deep breaths, using FIS and VIS in the order specified by the randomization, with an interval of at least 2 min between the devices. The average of 6 homogeneous respiratory cycles was considered for the data analysis performed by a bioengineer. The chest wall volumes and inspiratory muscle activity outcomes were assessed concurrently.

\section{Incentive Spirometers}

The studied FIS was the Respiron (NCS, São Paulo, Brazil). The studied FIS was the Voldyne 5000 (Hudson RCI/Teleflex Medical, Research Triangle Park, North Carolina). This choice was influenced by the fact that these devices are commonly used in our country. With the Respiron, during inspiration the patient makes the ball in the column rise and keeps it suspended with sustained inspiratory flow; this serves as visible feedback of the inspiratory flow. Similarly, with the Voldyne 5000 the patient makes a piston-plate rise and keeps it suspended.

The subject was instructed to perform a slow inhalation to raise the ball (FIS) or the piston-plate (VIS) and sustain the inflation for at least 5 seconds, followed by a normal exhalation. ${ }^{7,16}$ 


\section{Volume Rather Than Flow Incentive Spirometry}

\section{Thoracoabdominal Kinematics}

Thoracoabdominal kinematics were evaluated using optoelectronic plethysmography (OEP System, BTS Bioengineering, Garbagnate Milanese, Italy), as previously described. ${ }^{17}$ This equipment is based on 8 special video cameras (solid-state, charge-coupled devices) operating at 100 frames per second and synchronized with an infrared flashing light-emitting diode. Four cameras were positioned in front of the subject, and 4 behind. Eighty-nine retroreflective markers were placed on the anterior and posterior sides of the trunk, according to the protocol previously described by Aliverti et al. ${ }^{13}$ A 3-dimensional calibration of the equipment was performed, based on the manufacturer's recommendation. After that, the assessment was performed with the subject seated on a wheelchair without a back support, so the thoracoabdominal kinematics around the chest wall could be evaluated. The optoelectronic plethysmography software reconstructs the 3-dimensional position of each marker and computes the chest wall volumes with high accuracy. Algorithms computed the volume variations of the whole chest wall and the thoracic and abdominal compartments. The values for the upper and lower rib-cage and abdomen are expressed as absolute values and percentages.

\section{Respiratory Time Variables}

We calculated the mean inspiratory time $\left(\mathrm{T}_{\mathrm{I}}\right)$, total breathing cycle time $\left(\mathrm{T}_{\text {tot }}\right)$, and duty cycle $\left(\mathrm{T}_{\mathrm{I}} / \mathrm{T}_{\text {tot }}\right)$.

\section{Thoracoabdominal Asynchrony}

The thoracoabdominal asynchrony values were obtained using a calculation of the upper rib-cage to the abdominal phase angle, according to Agostoni et al. ${ }^{18}$ Phase angle was calculated as the lag time between the peaks of the upper rib-cage and the abdominal signals divided by the total cycle time times $360^{\circ}$.

\section{Respiratory Muscle Activity}

The activity of the sternocleidomastoid and external superior and inferior intercostal muscles was assessed using EMG signals (FreeEMG 300, BTS Bioengineering, Garbagnate Milanese, Italy) obtained simultaneously with the thoracoabdominal kinematics. Each EMG probe was attached to 2 reusable bipolar superficial electrodes consisting of $\mathrm{Ag} / \mathrm{AgCl}$ material and a conductive adhesive hydrogel (Maxicor, Brazil). The inter-electrode distance was $20 \mathrm{~mm}$. The skin was cleaned with an alcohol swab at all the attachment sites, to remove oils that decrease the impedance of the skin. Electrodes were affixed on the muscle belly, away from the motor point and parallel to the direction of the muscle fibers, per the European recommendations on noninvasive EMG. ${ }^{19}$ The right sternocleidomastoid electrode was placed on the muscle body, $5 \mathrm{~cm}$ from the mastoid process. ${ }^{20}$ For the external intercostal muscle of the right upper rib-cage, the electrode was placed on the second anterior intercostal space. ${ }^{21}$ For the external intercostal muscle of the left lower rib-cage, the electrode was placed on the 7th and 8th anterior intercostal spaces. ${ }^{21}$ All electrode positions were determined in accordance with the best signal capture, and the EMG analyses were carried out as recommended by Hermens et al. ${ }^{19}$

\section{Data Acquisition and Processing}

EMG signals were obtained using an 8-channel EMG module with wireless probes that had an acquisition frequency of $1,000 \mathrm{~Hz}$. Each probe consists of a mother electrode and a satellite electrode connected via a flexible cable, each fitted with a clip. The mother electrode contains an analog-to-digital converter with a resolution of 16 bits, an antenna, and a battery. The satellite electrode contains a signal-conditioning, low-pass filter with a frequency of $500 \mathrm{~Hz}$ and an amplifier with a gain range of $\pm 1.62 \mathrm{mV}$. All data were processed using dedicated software for acquisition and analysis (SMART, BTS Bioengineering, Garbagnate Milanese, Italy).

In the post-processing stage, we applied a Butterworth high-pass filter with a cutoff frequency of $20 \mathrm{~Hz}$; thus, the frequency range of the signal was set at $20-500 \mathrm{~Hz}$. To detect the linear envelope of the EMG signal, the signal was full-wave rectified and low-pass filtered. The electrical activity of the sternocleidomastoid and the upper and lower intercostal muscles was measured using the root mean square values and expressed in $10^{-3} \mathrm{mV}$.

\section{Data Analysis}

The sample size calculation was performed by considering the average difference of total chest wall volume generated by VIS relative to FIS as $475 \mathrm{~mL}$, with an average standard deviation of $15 \%(71 \mathrm{~mL})$ and a power of $80 \%$ as the primary variable. ${ }^{11}$ The sample size estimation was 16 subjects. Data values are presented as mean \pm SD. The differences between quiet breathing and breathing using the devices were analyzed using 1-way repeatedmeasures analysis of variance, with a post hoc Dunn test. The significance level was set to 5\%. The statistical analysis was performed using statistics software (SigmaStat 3.2, Systat Software, San Jose, California).

\section{Results}

Twenty-two subjects were screened; 20 met the eligibility criteria and were evaluated. A total of $12(60 \%)$ 


\section{Volume Rather Than Flow Incentive Spirometry}

Table. Respiratory Data and Muscular Activity During Quiet Breathing, Flow-Oriented Incentive Spirometry, and Volume-Oriented Incentive Spirometry

\begin{tabular}{|c|c|c|c|}
\hline & $\begin{array}{c}\text { Quiet } \\
\text { Breathing } \\
(\text { mean } \pm \text { SD) }\end{array}$ & $\begin{array}{l}\text { Flow-Oriented } \\
\text { Incentive Spirometry } \\
(\text { mean } \pm \text { SD) }\end{array}$ & $\begin{array}{l}\text { Volume-Oriented } \\
\text { Incentive Spirometry } \\
\quad(\text { mean } \pm \text { SD })\end{array}$ \\
\hline \multicolumn{4}{|l|}{ Volume, L } \\
\hline Chest wall & $0.62 \pm 0.18$ & $2.08 \pm 0.99 *$ & $2.48 \pm 1.22 * \dagger$ \\
\hline Upper rib cage & $0.22 \pm 0.11$ & $0.86 \pm 0.39 *$ & $0.97 \pm 0.57^{*}$ \\
\hline Lower rib cage & $0.12 \pm 0.07$ & $0.50 \pm 0.25^{*}$ & $0.57 \pm 0.36^{*}$ \\
\hline Abdomen & $0.28 \pm 0.09$ & $0.73 \pm 0.53 *$ & $0.94 \pm 0.55^{* \dagger}$ \\
\hline \multicolumn{4}{|l|}{ Time, s } \\
\hline Inspiratory & $2.00 \pm 0.81$ & $2.17 \pm 1.06$ & $3.81 \pm 3.30 * \dagger$ \\
\hline Total & $4.68 \pm 1.22$ & $4.94 \pm 2.49$ & $7.18 \pm 3.98 * \dagger$ \\
\hline Inspiratory/total & $0.43 \pm 0.10$ & $0.44 \pm 0.07$ & $0.42 \pm 0.11$ \\
\hline Asynchrony (upper rib cage $\times$ abdomen), phase angle & $7.08 \pm 9.28$ & $33.38 \pm 32.58$ & $14.48 \pm 14.70$ \\
\hline \multicolumn{4}{|l|}{ Root mean square muscle activity, $10^{-3} \mathrm{mV}$} \\
\hline Right sternocleidomastoid & $6.5 \pm 3.8$ & $22.8 \pm 20.4^{*} \dagger$ & $18.1 \pm 21.5^{*}$ \\
\hline Right intercostal & $6.5 \pm 1.6$ & $38.4 \pm 29.2 * \dagger$ & $27.0 \pm 24.1^{*}$ \\
\hline Left intercostal & $6.4 \pm 2.9$ & $16.4 \pm 9.8^{*}$ & $13.9 \pm 7.7^{*}$ \\
\hline
\end{tabular}

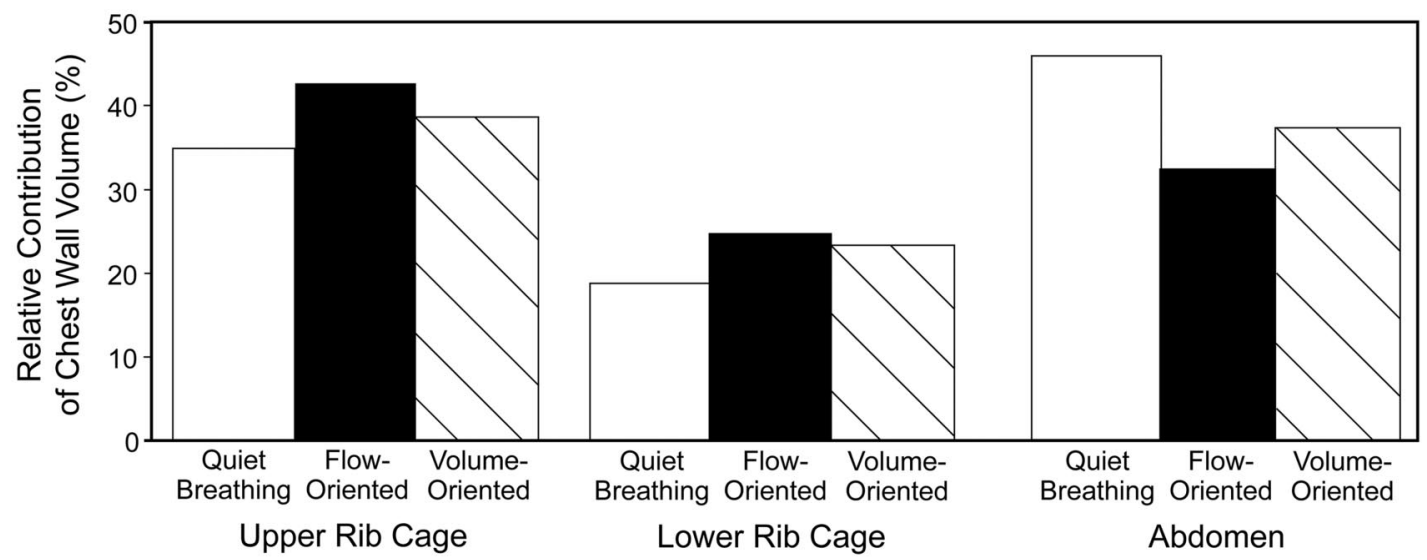

Fig. 1. Contribution of upper rib-cage, lower rib-cage, and abdominal motion to pulmonary volume during quiet breathing, flow-oriented incentive spirometry, and volume-oriented incentive spirometry.

subjects were females. The mean \pm SD age was $25.9 \pm$ 4.3 years, and the average body mass index was $23.6 \pm$ $2.4 \mathrm{~kg} / \mathrm{m}^{2}$. Their mean $\pm \mathrm{SD}$ lung function measurements were FVC $103.6 \pm 13.2 \%$ of predicted, $\mathrm{FEV}_{1} 101.4 \pm$ $12.7 \%$ of predicted, and $\mathrm{FEV}_{1} / \mathrm{FVC} 83.5 \pm 6.6 \%$.

\section{Thoracoabdominal Volumes}

Both FIS (335\%) and VIS (400\%) increased the chest wall volume compared to quiet breathing $(P<.001$, Table). However, the chest wall volume obtained with VIS was $65 \%$ greater than that obtained with FIS $(P=.007)$.

The FIS and VIS induced similar increases in the upper (138\% and $156 \%$, respectively) and lower respiratory com- partments (80\% and 91\%, respectively) compared to quiet breathing. In addition, FIS and VIS induced displacement in the abdominal compartment of $117 \%$ and $151 \%$, respectively, compared to quiet breathing $(P<.001$, see the Table and Fig. 1). However, VIS induced a $34 \%$ greater displacement, compared to FIS $(P=.03)$.

\section{Respiratory Time Variables}

There was an increase in $\mathrm{T}_{\mathrm{I}}$ and $\mathrm{T}_{\text {tot }}$ when using VIS, compared to using FIS $\left(\mathrm{T}_{\mathrm{I}} 3.81 \pm 3.30 \mathrm{~s}\right.$ vs $2.17 \pm 1.06 \mathrm{~s}$, and $\mathrm{T}_{\text {tot }} 7.18 \pm 3.98 \mathrm{~s}$ vs $4.94 \pm 2.49 \mathrm{~s}$, respectively) ( $P=.04$, see the Table). However, there was no difference 


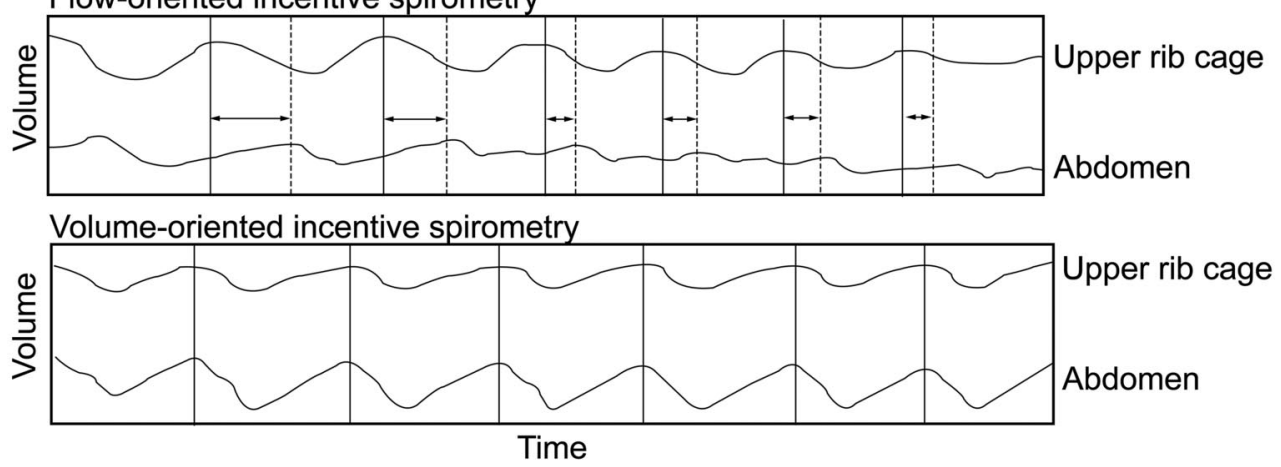

Fig. 2. Thoracoabdominal asynchrony during flow-oriented incentive spirometry versus volume-oriented incentive spirometry. LT = lag time between the end-inspiratory volume of the upper-rib-cage signal and the abdomen signal.

in $\mathrm{T}_{\mathrm{I}} / \mathrm{T}_{\text {tot }}$ between VIS and FIS $(0.42 \pm 0.11$ vs $0.44 \pm 0.07$, respectively) $(P=.64$, see the Table).

\section{Thoracoabdominal Asynchrony}

Asynchrony was observed when subjects used FIS, but not when they used VIS $(P=.03$, see the Table and Fig. 2).

\section{Electromyography}

The EMG of the right sternocleidomastoid, right upper rib-cage, and left lower rib-cage showed an increase in electrical activity when using FIS, compared to using VIS $(P<.001$, see the Table $)$.

\section{Discussion}

Our results show that VIS and FIS increased pulmonary volumes in healthy adults; however, VIS induced a greater total chest wall volume, especially in the abdominal compartment, and lower respiratory muscle activity, compared to FIS. Moreover, we observed that only FIS promoted thoracoabdominal asynchrony. To the best of our knowledge, this is the first study to compare both types of incentive spirometry using accurate equipment that allows chest wall volume assessment in a 3-dimensional and 3-compartment analysis that also simultaneously quantifies the respiratory muscle activity.

Although VIS or FIS are widely used and recommended in clinical practice, especially for perioperative care, there is no consensus about their benefits or indications, and no study has demonstrated which incentive spirometer is the most effective. ${ }^{22-24}$ This is most likely because few studies have assessed the differences in respiratory mechanics between the 2 devices. Parreira et al ${ }^{10}$ and Tomich et $\mathrm{al}^{11}$ evaluated thoracoabdominal motion when using FIS and VIS in healthy adults, and showed that VIS induced higher chest wall expansion, compared to FIS. Although these results appear similar to ours, they observed reduced volumes at baseline (tidal volume of $300 \mathrm{~mL}$ ) and during FIS $(1,264 \mathrm{~mL})$ and VIS $(1,739 \mathrm{~mL})$, compared with our results (respectively, $620 \mathrm{~mL}, 2,000 \mathrm{~mL}$, and 2,480 mL). There are at least 2 possible explanations for that discrepancy: differences in subject position when using VIS and FIS, and differences in the equipment used to evaluate thoracoabdominal mechanics.

In previous studies ${ }^{10,11}$ FIS and VIS were performed in a semi-reclined position $\left(45^{\circ}\right)$; however, several studies showed that chest wall volume and the relative contribution of the rib cage to tidal breathing are higher in spontaneous quiet breathing in the seated position, compared to the supine position. ${ }^{12,25}$ This is because the geometry of the respiratory muscles is strongly influenced by posture. For instance, the diaphragm has a reduced capacity to generate strength in the supine position. ${ }^{12}$ In addition, it is possible to generate higher chest wall volumes in the seated position without back support, ${ }^{12}$ which may explain the higher volumes observed in our study. Our use of optoelectronic plethysmography may also explain the higher chest wall volumes we obtained, because optoelectronic plethysmography demonstrates excellent consistency in estimating the lung volumes ${ }^{26}$ and allows the evaluation of thoracoabdominal motion in a 3-dimensional analysis. ${ }^{13,17}$ At this point it is not possible to determine if it was the subject's position or the use of the more precise technique (optoelectronic plethysmography) that was the main reason for the increased chest wall volume we observed. However, we believe that our findings are quite relevant, because seated incentive spirometry is more common in clinical practice. ${ }^{12}$

Interestingly, we also observed that VIS induced a greater abdominal displacement, and we hypothesize that this may have occurred because VIS is performed with lower inspiratory flow, which optimizes diaphragmatic excursion and improves the expansion of the basal area of the chest wall. Our data are supported by results obtained by Chuter 


\section{Volume Rather Than Flow Incentive Spirometry}

et al showing that FIS does not increase the abdominal contribution to total chest wall volume in patients who have had abdominal surgery. ${ }^{27}$

Our study also showed that FIS promoted thoracoabdominal asynchrony. Although the occurrence of asynchrony may seem unusual in healthy subjects, previous studies have observed thoracoabdominal asynchrony associated with increased respiratory loads in healthy subjects. ${ }^{28}$ Previous studies have also suggested that using FIS requires an increase in the activity of the respiratory muscles, compared to VIS. ${ }^{10}$ Based on those results, we suggest that FIS can impose an additional load on the respiratory system, leading to thoracoabdominal asynchrony. We also observed that VIS induced lower activity of the sternocleidomastoid and intercostal muscles, compared to FIS, suggesting that the lower effort of the inspiratory muscles may be due to increased displacement of the abdominal compartment (described above).

\section{Limitations}

First, the effect of incentive spirometry was evaluated in subjects with normal lung function; however, our research group is conducting studies with the same protocol in different populations, including the morbidly obese and elderly, to increase the practical applicability of the incentive spirometer.

Second, muscle activity was evaluated using surface EMG, and there is no consensus about electrode positioning for the respiratory muscles. In our study the electrodes were located according to previous studies performed by experts in this field. ${ }^{20,21}$

\section{Practical Implications}

Our findings show the acute effects of lung expansion using 2 types of incentive spirometer on healthy subjects; however, this suggests the need for a large, randomized controlled trial using incentive spirometry in clinical populations, and the evaluation of long-term effects of incentive spirometry on postoperative complications and hospital stay. Another important fact that must be considered in clinical practice is the difference in the cost of incentive spirometers (U.S.\$ 80 for VIS and U.S.\$ 20 for FIS).

\section{Conclusions}

The VIS promotes a greater chest wall volume with a higher abdominal contribution and lower muscle activity, without inducing thoracoabdominal asynchrony, compared to FIS, in healthy subjects.

\section{REFERENCES}

1. Agostini P, Cieslik H, Rathinam S, Bishay E, Kalkat MS, Rajesh PB, et al. Postoperative pulmonary complications following thoracic surgery: are there any modifiable risk factors? Thorax 2010;65(9): 815-818.

2. Pasquina P, Tramer MR, Granier JM, Walder B. Respiratory physiotherapy to prevent pulmonary complications after abdominal surgery: a systematic review. Chest 2006;130(6):1887-1899.

3. Suesada MM, Martins MA, Carvalho CR. Effect of short-term hospitalization on functional capacity in patients not restricted to bed. Am J Phys Med Rehabil 2007;86(6):455-462.

4. Schweinberger MH, Roukis TS. Effectiveness of instituting a specific bed protocol in reducing complications associated with bed rest. J Foot Ankle Surg 2010;49(4):340-347.

5. Carvalho CRF, Paisani DM, Lunardi AC. Incentive spirometry in major surgeries: a systematic review. Braz J Physiother 2011;15(5): 343-350.

6. Guimarães MM, El Dib R, Smith AF, Matos D. Incentive spirometry for prevention of postoperative pulmonary complications in upper abdominal surgery. Cochrane Database Syst Rev 2009;(3):CD006058.

7. Restrepo RD, Wettstein R, Wittnebel L, Tracy M. AARC Clinical Practice Guidelines. Incentive spirometry: 2011. Respir Care 2011; 56(10):1600-1604.

8. Pasquina $P$, Tramèr MR, Walder B. Prophylactic respiratory physiotherapy after cardiac surgery: systematic review. BMJ 2003;327(7428): 1379-1384.

9. Agostini P, Calvert R, Subramanian H, Naidu B. Is incentive spirometry effective following thoracic surgery? Interact Cardiovasc Thorac Surg 2007;7(2):297-300.

10. Parreira VF, Tomich VM, Brito RR, Sampaio RF. Assessment of tidal volume and thoracoabdominal motion using volume and floworiented incentive spirometers in healthy subjects. Braz J Medical Biol Res 2005;38(7):1105-1112.

11. Tomich GM, França DC, Diório ACM, Brito RR, Sampaio RF, Parreira VF. Breathing pattern, thoracoabdominal motion and muscular activity during three breathing exercises. Braz J Med Biol Res 2007;40(10):1409-1417.

12. Romei M, Lo Mauro A, Turconi AC, Bresolin N, Pedotti A, Aliverti A. Effects of gender and posture on thoracoabdominal kinematics during quiet breathing in healthy adults. Respir Physiol Neurobiol 2010;172(3):184-191.

13. Aliverti A, Quaranta M, Chakrabarti B, Albuquerque ALP, Calverley PM. Paradoxical movement of the lower ribcage at rest and during exercise in COPD patients. Eur Respir J 2009;33(1):49-60.

14. Vogiatzis I, Aliverti A, Golemati S, Georgiadou O, Lomauro A, Kosmas E, et al. Respiratory kinematics by optoelectronic plethysmography during exercise in men and women. Eur J Appl Physiol 2005;93(5-6):581-587.

15. Miller MR, Hankinson J, Brusasco V, Burgos F, Casaburi R, Coates A, et al; ATS/ERS Task Force. Standardisation of spirometry. Eur Respir J 2005;26(2):319-338.

16. Sarro KJ, Silvatti AP, Aliverti A, Barros RML. Proposition and evaluation of a novel method based on videogrammetry to measure three-dimensional rib motion during breathing. J Appl Biomech 2009; 25(3):247-252.

17. Aliverti A, Carlesso E, Raffaele Dellacà R, Pelosi P, Chiumello D, Pedotti A, Gattinoni L. Chest wall mechanics during pressure support ventilation. Crit Care 2006;10(2):1-10.

18. Agostoni E, Mognoni P. Deformation of the chest wall during breathing efforts. J Appl Physiol 1966;21(6):1827-1832.

19. Hermens HJ, Freriks B, Disselhorst-Klug C, Rau G. Development of recommendations for SEMG sensors and sensor placement procedures. J Electromyogr Kinesiol 2000;10(5):361-374. 


\section{Volume Rather Than Flow Incentive Spirometry}

20. Kallenberg LA, Preece S, Nester C, Hermens HJ. Reproducibility of MUAP properties in array surface EMG recordings of the upper trapezius and sternocleidomastoid muscle. J Electromyogr Kinesiol 2009;19(6):536-542.

21. Maarsingh EJ, van Eykern LA, Sprikkelman AB, Hoekstra MO, van Aalderen WM. Respiratory muscle activity measured with a noninvasive EMG technique: technical aspects and reproducibility. J Appl Physiol 2000;88(6):1955-1961.

22. Smetana GW, Lawrence VA, Cornell JE. Preoperative pulmonary risk stratification for noncardiothoracic surgery: systematic review for the American College of Physicians. Ann Intern Med 2006; 144(8):581-595.

23. Arozullah AM, Conde MV, Lawrence VA. Preoperative evaluation for postoperative pulmonary complications. Med Clin N Am 2003; 87(1):153-173.
24. Overend TJ, Anderson CM, Lucy SD, Bhatia C, Jonsson BI, Timmermans $\mathrm{C}$. The effect of incentive spirometry on postoperative pulmonary complication: a systematic review. Chest 2001;120(3):971-978.

25. Lee LJ, Chang AT, Coppieters MW, Hodges PW. Changes in sitting posture induce multiplanar changes in chest wall shape and motion with breathing. Respir Physiol Neurobiol 2010;170(3):236-45.

26. Cala SJ; Kenyon CM Ferrigno G; Carnevali P; Aliverti A, Pedotti. A 1996. Chest wall and lung volume estimation by optical reflectance motion analysis. J Appl Physiol 1996;81(6):2680-2689.

27. Chuter TAM, Weissman C, Starker PM, Gump FE. Effect of incentive spirometry on diaphragmatic function after surgery. Surgery 1989;105(4):488-493.

28. Brack T, Jubran A, Tobin MJ. Effect of resistive loading on variation activity of breathing. Am J Crit Care Med 1998;157(6 Pt 1):17561763. 